\title{
Human epithelial cells establish direct antifungal defense through TLR4-mediated signaling
}

\author{
Günther Weindl, ${ }^{1,2}$ Julian R. Naglik, ${ }^{3}$ Susanne Kaesler, ${ }^{1}$ Tilo Biedermann, ${ }^{1}$ Bernhard Hube, ${ }^{4}$ \\ Hans Christian Korting, ${ }^{2}$ and Martin Schaller ${ }^{1,2}$

\begin{abstract}
${ }^{1}$ Department of Dermatology, Eberhard Karls University of Tübingen, Tübingen, Germany. ${ }^{2}$ Department of Dermatology, Ludwig Maximilians University of Munich, Munich, Germany. ${ }^{3}$ Departments of Oral Medicine, Pathology, and Immunology, King's College London Dental Institute, London, United Kingdom. ${ }^{4}$ Department of Microbial Pathogenicity Mechanisms,

Leibniz Institute for Natural Product Research and Infection Biology, Hans Knöll Institute and Friedrich Schiller University Jena, Jena, Germany.
\end{abstract}

\begin{abstract}
Mammalian TLRs are central mediators of the innate immune system that instruct cells of the innate and adaptive response to clear microbial infections. Here, we demonstrate that human epithelial TLR4 directly protected the oral mucosa from fungal infection via a process mediated by polymorphonuclear leukocytes (PMNs). In an in vitro epithelial model of oral candidiasis, the fungal pathogen Candida albicans induced a chemoattractive and proinflammatory cytokine response but failed to directly modulate the expression of genes encoding TLRs. However, the addition of PMNs to the C. albicans-infected model strongly upregulated cytoplasmic and cellsurface epithelial TLR4 expression, which correlated directly with protection against fungal invasion and cell injury. C. albicans invasion and cell injury was restored by the addition of TLR4-specific neutralizing antibodies and knockdown of TLR4 using RNA interference, even in the presence of PMNs, demonstrating the direct role of epithelial TLR4 in the protective process. Furthermore, treatment with neutralizing antibodies specific for TNF- $\alpha$ resulted in strongly reduced TLR 4 expression accompanied by augmented epithelial cell damage and fungal invasion. To our knowledge, this is the first description of such a PMN-dependent, TLR4-mediated protective mechanism at epithelial surfaces, which may provide significant insights into how microbial infections are managed and controlled in the oral mucosa.
\end{abstract}

\section{Introduction}

The mucosal epithelium has immense importance in host defense and immune surveillance, because it is the primary cell layer that initially encounters the majority of microorganisms. This specialized interaction will result in either passive coexistence between microbe and host, as in the case of commensal microbes, or a breach of the mucosal barrier and subsequent cell injury, as in the case of microbial pathogens (1). Barrier function alone is usually adequate to restrain commensal microbes, but is often insufficient to protect against microbial pathogens. Accordingly, the oral epithelium is able to secrete a variety of defense effector molecules and to orchestrate an immune inflammatory response to activate myeloid cells in the submucosal layers to clear any invading pathogens $(2,3)$.

Immune responsiveness to many microbial pathogens depends on a family of pattern recognition receptors known as TLRs, which are the major innate recognition system for microbial invaders in vertebrates (4). Ten TLR members exist in humans; these are triggered by conserved molecular structures (pathogen-associated molecular patterns) expressed by bacteria, viruses, and fungi. These include LPS, peptidoglycan, lipoprotein moieties, protein motifs, and nucleotide sequences $(4,5)$. However, in addition to their function in host defense, recent findings indicate that TLRs

Nonstandard abbreviations used: $\mathrm{LDH}$, lactate dehydrogenase; $\mathrm{MCP}$, monocyte chemoattractant protein; MIP, macrophage inflammatory protein; PMN, polymorphonuclear leukocyte; RHE, reconstituted human epithelium.

Conflict of interest: The authors have declared that no conflict of interest exists. Citation for this article: J. Clin. Invest. 117:3664-3672 (2007). doi:10.1172/JCI28115. also appear to have a more general role in epithelial homeostasis and protection from cell injury (6).

Candida albicans is a ubiquitous commensal organism and the most common fungal pathogen of humans and accounts for more than $50 \%$ of all fungal systemic infections $(7,8)$. Host defense mechanisms against mucosal candidiasis are not well understood, but include both innate and adaptive responses. Both TLR2 and TLR4 have been implicated in host defense against C. albicans (5); however, the majority of these studies have been based on TLR recognition by myeloid cells and thus indicate a general role for the TLRs in systemic candidiasis $(9,10)$. A great deal less is known about interactions of TLRs with Candida at mucosal surfaces. Several TLRs are expressed constitutively in the oral epithelium (11), and increased expression of TLR2 and TLR4 has previously been observed in inflamed gingival epithelial tissues (12). C. albicans is able to activate NF- $\mathrm{KB}$ in epidermal keratinocytes, the main transcriptional factor associated with TLR signaling, and can also stimulate the production of IL-8 (13), a powerful chemokine involved in recruitment of polymorphonuclear leukocytes (PMNs) to sites of microbial infection $(1,4,14)$.

PMNs represent a central component of the innate immune response (15). In many mucosal infections and inflammatory disorders, the combination of epithelial injury, disease activity, and symptoms parallel PMN infiltration of the mucosa $(16,17)$. Similarly, during oral Candida infections, transepithelial migration of PMNs is believed to play a crucial role in the clearance of infection and in epithelial homeostasis (18). Previously, using a model of oral reconstituted human epithelium (RHE), we demonstrated that PMNs could protect the epithelium from C. albicans-induced 


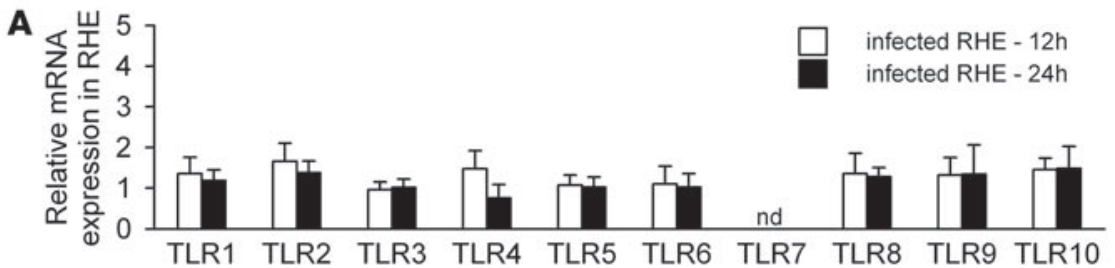

B

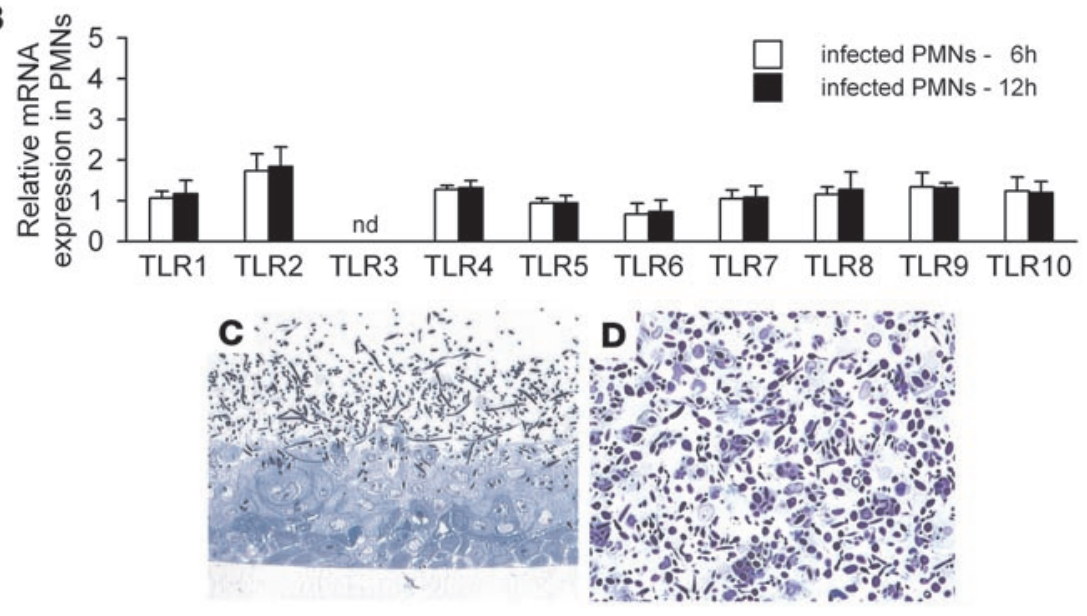

\section{Figure 1}

Interaction of oral RHE and PMNs with C. albicans does not alter the constitutive levels of TLR1-10 expression. (A and B) TLR1-TLR10 mRNA levels in C. albicans-infected $\operatorname{RHE}(\mathbf{A}, n=6)$ and after coincubation of PMNs with C. albicans (B, $n=6)$. Values are normalized to housekeeping genes YWHAZ and G6PD and relative to uninfected RHE or PMNs (control, assigned as 1.0). nd, not detected. (C and D) Light micrographs of oral RHE $24 \mathrm{~h}$ after $\mathrm{C}$. albicans infection (C) and of PMNs $12 \mathrm{~h}$ after coincubation with $C$. albicans (D). Original magnification, $\times 40$. cell injury via a process that was independent of phagocytosis, PMN transmigration, or even physical PMN-epithelial cell contact (19). This protective phenotype was associated with the production of epithelial IL-8 and GM-CSF, as well as other proinflammatory cytokines including IL- $1 \alpha$ and $\beta$, IL- 6 , and TNF- $\alpha$. These cytokines have been implicated in the development of protective immunity against systemic Candida infections (20). However, the mechanism by which PMNs and epithelial cells interact to protect the mucosal surfaces from microbial invasion is as yet unclear. Our study aimed to resolve this key issue by addressing 2 fundamental questions that would significantly enhance our understanding of this primary defense mechanism. Do PMNs protect the oral mucosa from fungal infection directly or indirectly through epithelial cells? And do epithelial TLRs orchestrate the antifungal protective response?

Here we report that immunological crosstalk between C. albicans-infected oral epithelium and PMNs caused PMN-mediated upregulation of epithelial TLR4. Furthermore, we demonstrate that epithelial TLR4 was directly responsible for protecting the mucosal surface from fungal invasion and cell injury and that TNF- $\alpha$, among other Candida-induced cytokines, played an essential role in this 3-way communication mechanism.

\section{Results}

$T L R$ gene expression in oral RHE is representative of the oral TLR expression profile in vivo. Previously, we have used the oral RHE models to study a number of different aspects of host-Candida interactions $(19,21-23)$. In this study, we used the oral RHE model to investigate the role of human TLRs in protection against Candida infection. To do this, we first needed to ensure that the TLR profiles in the oral RHE models mimic the TLR profiles in vivo. Real-time RT-PCR demonstrated a high degree of similarity in TLR expression profiles between the oral RHE (see below) and buccal epithelial samples isolated from 17 healthy individuals. In the oral RHE model, we demonstrated constitutive expression of all the TLRs except TLR7 at a low level (Supplemental Figure 1; supple- mental material available online with this article; doi:10.1172/ JCI28115DS1). Similarly, in samples from healthy individuals, all the TLRs except TLR5 and TLR7 were detected. TLR3, TLR6, TLR9, and TLR10 are expressed in buccal cells, albeit at low levels. The most commonly expressed TLR genes in vivo were TLR1 (82\%), TLR2 (100\%), TLR4 (88\%), and TLR8 (79\%), with TLR1 being the most highly expressed gene (Supplemental Figure 1). TLR1 was also the most highly expressed TLR gene in RHE. All TLR expression data were of epithelial origin, because the presence of transcripts characteristic for neutrophils (CD66b and myeloperoxidase), dendritic cells (CD11c), and T cells (CD3) were not detected by TaqMan real-time RT-PCR in any of the human in vivo RNA samples (data not shown). Our results indicated that the oral RHE TLR profiles were representative of the in vivo situation and could be used to study epithelial TLR interactions with human pathogens.

C. albicans does not alter constitutive TLR expression in oral epithelium or $P M N s$. To establish the baseline characteristics of our PMN-supplemented oral RHE model system, we determined the TLR1-TLR10 expression profiles in the oral RHE model (see above) and in PMNs separately. In unstimulated human PMNs we confirmed previous results (24) by detecting mRNA transcripts for all TLRs except TLR3 (data not shown). Exposure of the oral RHE to C. albicans for 12 or $24 \mathrm{~h}$ and of PMNs to C. albicans for 6 or $12 \mathrm{~h}$ failed to modulate TLR mRNA expression levels, despite clear evidence of fungal infection and epithelial cell injury by light microscopy (Figure 1).

PMNs stimulate epithelial TLR4 gene upregulation in the presence of C. albicans. PMNs are frequently found at sites of Candida infections and are thought to be crucial players in controlling oral disease in vivo $(25,26)$. We have previously demonstrated that addition of PMNs to the C. albicans-infected RHE model can protect the oral epithelium from fungal invasion and cell damage (19). In the present study, we investigated whether this $\mathrm{PMN}$-mediated protective phenotype was associated with TLR responses. Upon PMN supplementation to the C. albicans-infected oral RHE, we observed a 30 -fold increase of epithelial TLR4 mRNA after $12 \mathrm{~h}$ and a striking 

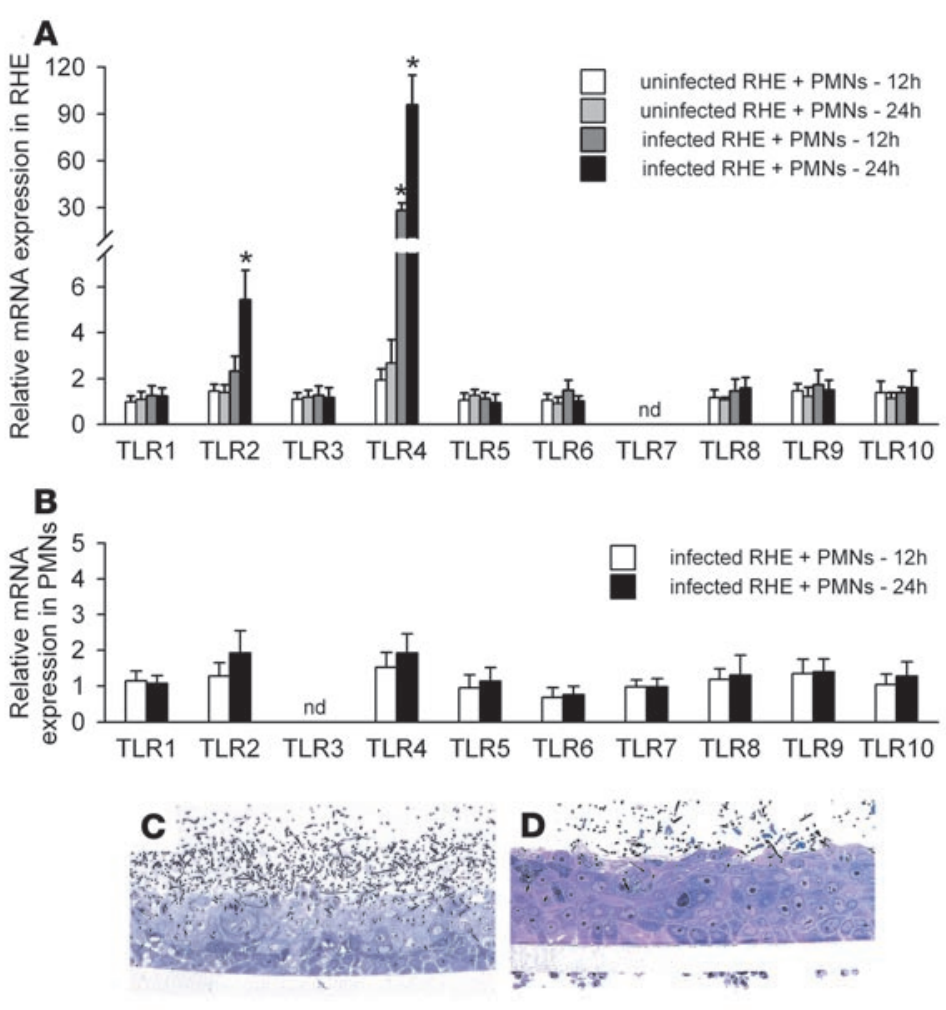

100-fold upregulation after $24 \mathrm{~h}$ (Figure 2A). A small but significant increase of TLR 2 mRNA levels was found after $24 \mathrm{~h}$, but no change in expression of the other TLR mRNAs was noted. No significant epithelial TLR4 upregulation was observed in the absence of C. albicans (Figure 2A) or in the C. albicans-infected oral RHE without PMNs (Figure 1A). Furthermore, TLR4 upregulation was observed with heat-killed C. albicans, which suggests that cell injury or the exposure of deeper epithelial layers to C. albicans cells is not required to trigger PMN-mediated TLR4 upregulation (data not shown). In addition, after infection of the oral RHE with less virulent Candida species (C. glabrata and C. tropicalis), only a modest increase in TLR4 mRNA expression was evident in the presence of PMNs (Supplemental Figure 2), indicating that epithelial cells might recognize certain cell surface moieties of $C$. albicans that are less abundant or structurally different in non-C. albicans species. TLR4 expression was of epithelial origin, because no changes in TLR mRNA levels were detected in the PMNs after their removal from the basal side of the filter at the same time points (Figure 2B). Histological analysis revealed that epithelial TLR4 upregulation coincided with a significant reduction in fungal invasion and epithelial cell injury 6 and 12 h after PMN addition compared with the PMN-absent control (Figure 2, C and D). These marked histological differences in the presence of PMNs corresponded to a significant decrease in lactate dehydrogenase (LDH) release, which is used as a marker of epithelial cell damage (data not shown). Furthermore, this PMNmediated protective phenotype was observed using different PMN donors and PMN pools (data not shown), demonstrating the universal protective nature of PMNs against mucosal fungal infection. Together, the data indicate what we believe to be a novel 3-way communication system among C. albicans, oral epithelium, and PMNs that results in the PMN-mediated upregulation of epithelial TLR4 and concomitant protection against fungal infection.

\section{Figure 2}

PMNs induce epithelial TLR4 gene upregulation, which protects against fungal invasion and cell damage, in the presence of $C$. albicans. (A) TLR expression in uninfected and infected oral $\mathrm{RHE}$ in the presence of PMNs. Expression values are normalized to YWHAZ and G6PD and relative to uninfected RHE without PMNs (control, assigned as 1.0). ${ }^{\star} P<0.05$, 2-tailed paired Student's $t$ test. (B) TLR expression in the PMNs isolated from the filter of the $C$. albicans-infected $\mathrm{RHE}$, relative to PMNs isolated from uninfected RHE (control, assigned as 1.0) and normalized to YWHAZ $(n=6)$. (C and D) C. albicans-infected oral RHE after $24 \mathrm{~h}$. In the absence of PMNs, extensive edema and vacuolization was observed (C), while a strongly reduced fungal virulence phenotype was evident following PMN addition to the basal side of the filter after $12 \mathrm{~h}$ (D). Original magnification, $\times 40$.

PMNs stimulate TLR4 protein expression in epithelial cells in direct contact with C. albicans. To confirm our gene expression results on the protein level and to determine the localization of TLR4 upregulation, we performed confocal laser and immunoelectron microscopy. Corresponding to the RT-PCR data, we observed a weak but equal distribution of constitutive TLR4 expression in all epithelial layers of the uninfected RHE in the presence and absence of PMNs (Figure 3, A and B). C. albicans infection without PMNs did not markedly alter TLR4 protein expression at the apical epithelial layers directly in contact with the fungus (Figure 3C). However, when PMNs were added to the basal side of the C. albicans-infected oral RHE, a strong increase in epithelial TLR4 protein expression was observed in the epithelial layers (Figure 3D). Fluorescence intensity profiles of these images confirmed increased TLR4 expression in the presence of PMNs (Supplemental Figure 3). At a higher magnification, the upregulation in TLR4 expression was observed to be localized primarily within the cytoplasm of oral epithelial cells (Figure 3E). This is in accordance with previous studies showing that TLR4 is generally localized to the Golgi apparatus of intestinal epithelial cells $(2,27)$ and indicates that responsiveness of the epithelium may involve some sort of cellular processing and transport (14). Immunoelectron microscopy revealed TLR4 expression on the cell surface at the site of direct host-pathogen contact (Figure 4, A and B) and also confirmed the confocal data (Figure 4, $\mathrm{C}$ and D) showing TLR4 expression within the cytoplasm.

Epithelial TLR4 protects the oral mucosa from fungal invasion and cell injury. Our data indicated that PMNs mediate both the upregulation of epithelial TLR4 and the concomitant protection against C. albicans infection. However, we were still uncertain as to whether epithelial TLR4 was directly responsible for protecting the oral mucosa from fungal invasion.

To test this, the oral RHE samples were preincubated with neutralizing TLR $4 \mathrm{mAbs} 1 \mathrm{~h}$ prior to C. albicans inoculation and supplemented with PMNs 12 h later (blocking TLR2 antibodies and isotype-matched antibody IgG2a were also included as negative controls). At $24 \mathrm{~h}$, histological analysis revealed that in the presence of blocking TLR4 antibody C. albicans-induced tissue damage was restored (virulence phenotype), while isotype-matched control antibodies showed no effect (protective phenotype retained) (Figure 5, A-D). Histological data were supported by cell damage assays, which demonstrated that anti-TLR4 blocking antibodies significantly increased LDH release while the TLR2 blocking and 

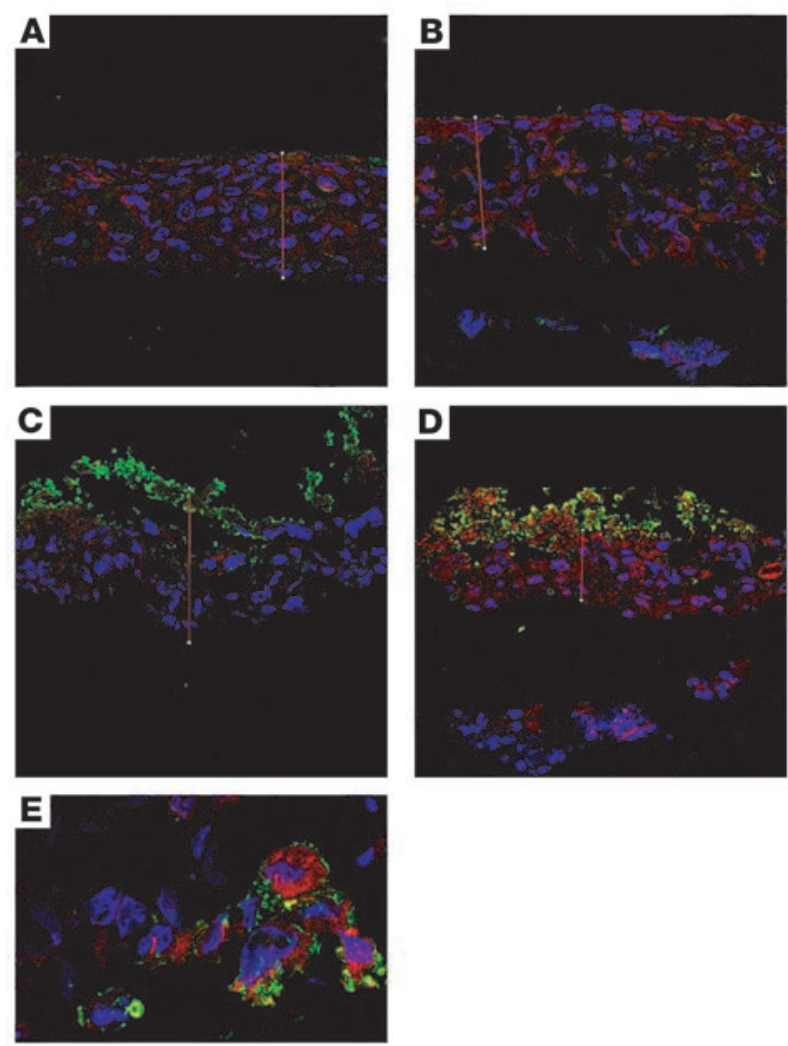

isotype-matched control antibodies did not (Figure 5E). The functional activity of anti-TLR2 antibodies was confirmed in Zymosan-treated PMNs, which showed decreased IL-8 release after neutralization of TLR2 (data not shown). Furthermore, real-time RT-PCR confirmed that TLR4 blocking antibodies were able to prevent TLR4 gene upregulation for the entire 24-h time course in all experiments (data not shown). It should be noted that when the C. albicans-infected oral RHE was supplemented with PMNs, the $\mathrm{LDH}$ values in the culture medium remained relatively high $(\sim 140$ U/l) compared with the PBS control without C. albicans $(\sim 20$ $\mathrm{U} / \mathrm{l}$ ) (Figure 5E). This was because the PMNs were added $12 \mathrm{~h}$ after C. albicans inoculation, thus allowing the fungus to induce cell damage in the initial 12 -h period. However, after PMN supplementation (12-24 h), no further increase in LDH value was detected; this effect was reversed after the addition of TLR4 blocking antibody, which restored tissue damage (Figure 5F). These data demonstrate that PMN addition induces an immediate protective effect on the oral epithelium and implicates epithelial TLR4 in directly protecting the oral mucosa against fungal invasion and cell injury.

\section{Figure 4}

Cell surface TLR4 directly recognizes $C$. albicans and is expressed intracellularly. Electron microscopy with postembedding immunogold labeling in C. albicans-infected oral RHE at $24 \mathrm{~h}$ in the presence of PMNs. (A) Expression of TLR4 was evident at the site of direct hostpathogen contact. (B) Higher-magnification view of boxed region in A. (C) An epithelial cell inside the oral RHE. (D) Higher-magnification view of boxed region in C revealed expression of TLR4 at the cell membrane and intracellularly. Arrows indicate gold-labeled TLR4 expression. Data are representative of 3 independent experiments. Original magnification, $\times 20,000(\mathbf{A}$ and $\mathbf{B}) ; \times 3,000(\mathbf{C}$ and $\mathbf{D})$.

\section{Figure 3}

PMNs stimulate TLR4 protein expression in epithelial cells in direct contact with $C$. albicans. Confocal laser microscopy of oral RHE after $24 \mathrm{~h}$ in the presence and absence of $C$. albicans and PMNs (cell nuclei, blue; TLR4, red; C. albicans, green). Basal expression (A) of TLR4 in the oral RHE was nearly unchanged after the addition of PMNs alone (B) or C. albicans alone (C). (D) Strong upregulation of TLR4 was clearly evident in the $C$. albicans-infected oral RHE in the presence of PMNs. (E) Higher-magnification image demonstrating increased TLR4 expression only in epithelial cells in direct contact with $C$. albicans in the presence of PMNs. Data are representative of 3 independent experiments. Original magnification, $\times 40$ (A-D); $\times 250$ (E).

Knockdown of epithelial TLR4 by RNA interference restores fungal invasion and cell injury. To confirm that epithelial TLR4 was directly responsible for this protective phenotype, we used RNA interference to knock down epithelial TLR4 expression in the presence of PMNs. Because the oral RHE models have not previously been used to our knowledge in RNA interference studies, in initial experiments we aimed to establish how effective siRNAs were at knocking down mRNA expression in this 3-dimensional organotypic system. Using fluorescently labeled nonsilencing control siRNA, only the uppermost apical epithelial cell layers appeared to be successfully transfected, whereas the remaining layers of the RHE showed no fluorescence (data not shown), indicating successful but limited penetration of the oral RHE. Hence, when quantified by real-time RT-PCR, there was a limited reduction in TLR4 mRNA levels by $75 \%$ because the whole of the RHE was removed for subsequent expression analyses (data not shown). Similarly, confocal microscopy images showed an approximately $60 \%$ reduction in epithelial TLR4 expression after transfection with TLR4 siRNA for $24 \mathrm{~h}$ (data not shown). However, in our experimental system there may not be a requirement for a complete knockout of TLR4 throughout the RHE, because TLR4 was almost exclusively upregulated in epithelial cells in the uppermost cell layers directly interacting with the fungal cells (Figure 3D). Thus, knockdown of TLR4 mRNA in the surface apical layers of the oral RHE may be sufficient to obtain a protective effect against C. albicans infection.
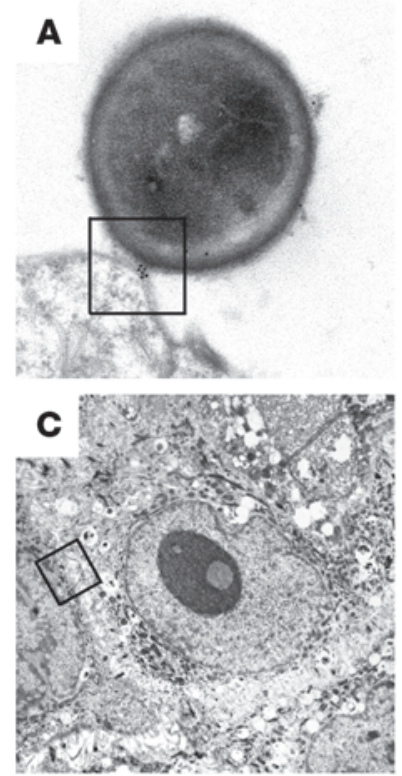
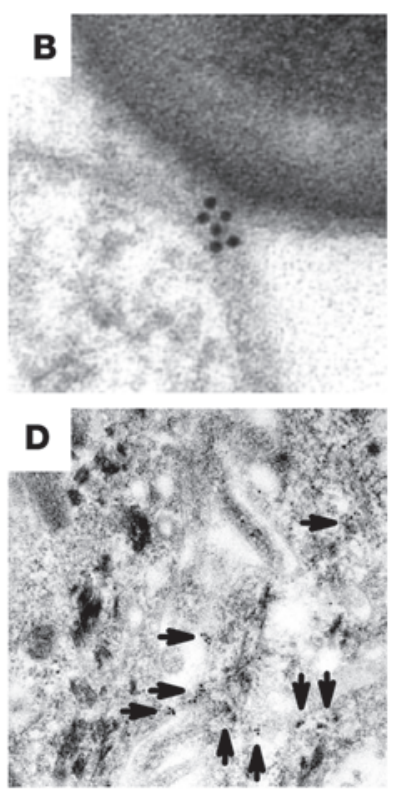

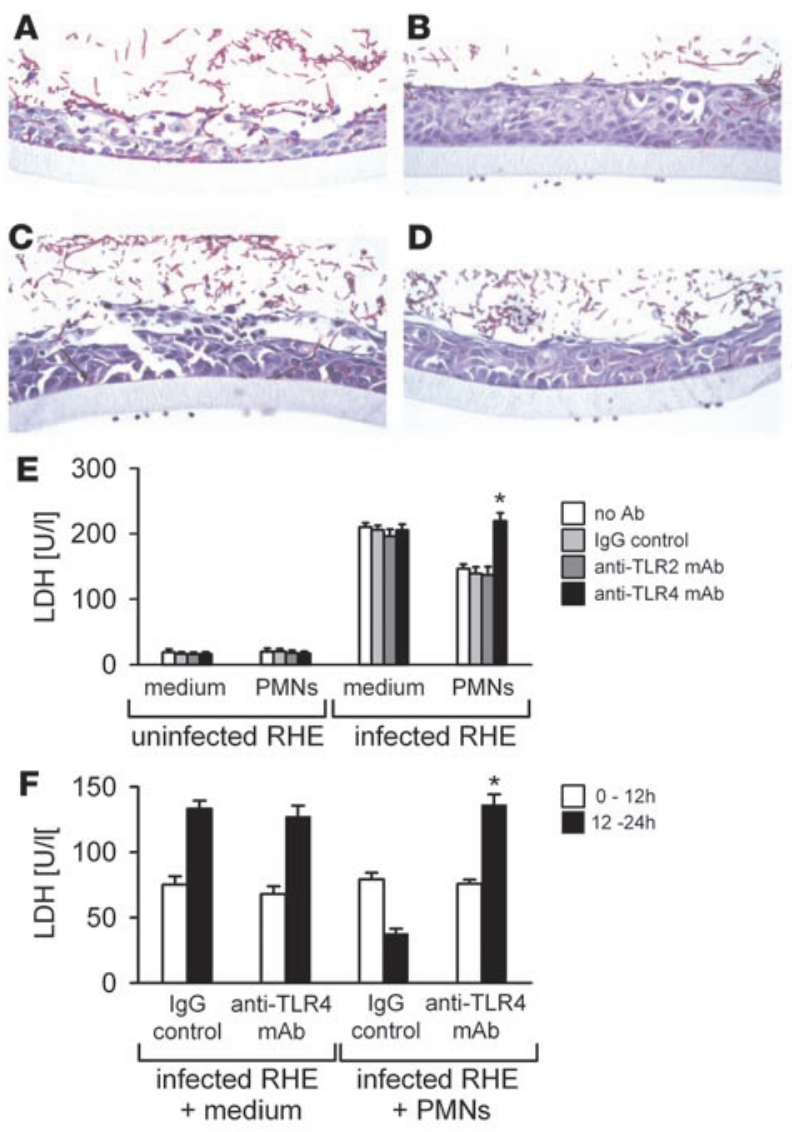

To test this, we transfected the oral RHE with 2 separate siRNAs to knock down TLR4 expression. Quantitative RT-PCR analysis demonstrated that TLR4 siRNA transfection prevented TLR4 upregulation in the infected RHE supplemented with PMNs (data not shown). Furthermore, quantitative analysis of images obtained by confocal microscopy demonstrated a $65 \%$ decrease in TLR4 immunofluorescence (Figure 6, A-C). Knockdown of TLR4 expression correlated with increased epithelial damage compared with control siRNA and samples without siRNAs (Figure 6D). Moreover, the addition of TLR4 siRNAs abrogated the protective effect of PMNs, because C. albicans-induced epithelial cell damage was restored after PMN addition (12-24 h; Figure 6E). These siRNA data paralleled the TLR4 blocking antibody data (Figure 5) and strongly support a direct role for epithelial TLR4 in the PMNmediated process that protects the oral mucosa from fungal invasion and cell injury.

Role of epithelial cytokines in the PMN-mediated TLR4-dependent protection mechanism. Given that cell-cell contact between epithelial cells and PMNs is prevented by the filter barrier, we investigated the role of epithelial chemokines and cytokines in initiating the protective response. Protein secretion and gene expression was quantified in the presence and absence of C. albicans, PMNs, TLR4 blocking antibodies, and TLR4 siRNAs by use of ELISA and realtime RT-PCR. In response to C. albicans (in the absence or presence of PMNs), epithelial cells secreted significantly increased concentrations of IL- 8 and GM-CSF $(P<0.05$; Supplemental Figure 4A); moderately increased levels of IL- $1 \alpha$, IL-1 $\beta$, IL-6, and TNF- $\alpha$; and unchanged levels of IL-2, IL-4, IL-12, and G-CSF (data not shown); which is in line with our previous study (19). Introduction of anti-

\section{Figure 5}

TLR4 blocking antibody reverses the antifungal protective effect of PMNs on the oral mucosa. (A-D) Light micrographs of RHE $24 \mathrm{~h}$ after infection with C. albicans SC5314 in the absence and presence of PMNs. (A) Invasion of $C$. albicans with extensive edema and vacuolization of all epithelial layers in the absence of PMNs. (B) Strongly reduced virulence phenotype of $C$. albicans at the same time point when PMNs were added after $12 \mathrm{~h}$ to the basal side of the filter. $(\mathbf{C})$ In the presence of anti-TLR4 neutralizing mAbs, C. albicans-induced tissue damage was restored, while (D) isotype-matched control IgG antibodies showed no effect on C. albicans virulence. PMNs were attached to the basal side of the filter in B-D. Data are representative of 3 experiments. Original magnification, $\times 40$. (E) Release of LDH by epithelial cells $24 \mathrm{~h}$ after infection (or not) with $C$. albicans in the presence and absence of PMNs (added after $12 \mathrm{~h})(n=6)$. (F) LDH values before $(0-12 \mathrm{~h})$ and after $(12-24 \mathrm{~h})$ addition of PMNs to $C$. albicans-infected RHE model $(n=4)$. ${ }^{\star} P<0.05$, 2-tailed paired Student's $t$ test.

TLR4 blocking antibodies or TLR4 siRNAs resulted in a moderate but significant downregulation of IL-8 production at $24 \mathrm{~h}$ $(P<0.05)$, but not of GM-CSF (Supplemental Figure 4, A and C). To ensure that the cytokine response was derived from the epithelium and not from the PMNs attached to the basal side of the filter, the increase in epithelial IL-8 and GM-CSF protein expression was confirmed at the mRNA level by real-time RT-PCR (Supplemental Figure 4B). The data thus indicate that C. albicans strongly stimulates IL-8 and GM-CSF secretion in epithelial cells and that the initial IL-8 response may be partially dependent upon TLR4.

IL- 8 and GM-CSF are key cytokines in recruiting and stimulating PMNs to sites of microbial infection $(1,4,14)$, which are rapidly activated by exposure to proinflammatory cytokines including IL- $1 \alpha$, IL-1 $\beta$, IL- 6 , and TNF- $\alpha$ (28). Together with the observation that epithelial macrophage inflammatory protein-1 $\beta$ (MIP-1 $\beta$ ) and monocyte chemoattractant protein-1 (MCP-1) production was also increased after PMN supplementation of the C. albicansinfected oral RHE model, we investigated whether blocking of these 8 cytokines with neutralizing antibodies could prevent epithelial TLR4 upregulation and subsequent protection. Incubation of the RHE with neutralizing antibodies had a minimal effect on epithelial cell damage, and strong TLR4 upregulation was still induced after PMN addition (protective phenotype; data not shown). Interestingly, incubation of PMNs with all neutralizing antibodies, except GM-CSF, led to strongly reduced TLR4 mRNA expression (data not shown). The strongest effect was observed after neutralization of TNF- $\alpha$ (Figure 7A). Analysis of TLR4 protein expression by confocal microscopy showed a similar decrease (Figure 7, B-D), with strongly reduced expression in the basal epithelial cell layer (Figure 7C). Furthermore, compared with control antibodies, neutralization of TNF- $\alpha$ led to increased epithelial damage and abolished the protective effect of PMNs, as indicated by increased LDH release (12-24 h) after addition of PMNs (Figure 7E).

\section{Discussion}

TLR recognition of pathogenic microbes at mucosal surfaces orchestrates innate immune responses through the induction of chemokines and inflammatory cytokines, which coordinate the recruitment of PMNs and activation of macrophages that in turn leads to direct killing of the invading pathogens, primarily by phagocytosis $(1,4,14)$. Our data demonstrate that protection against fungal invasion and cell injury can also be achieved with- 

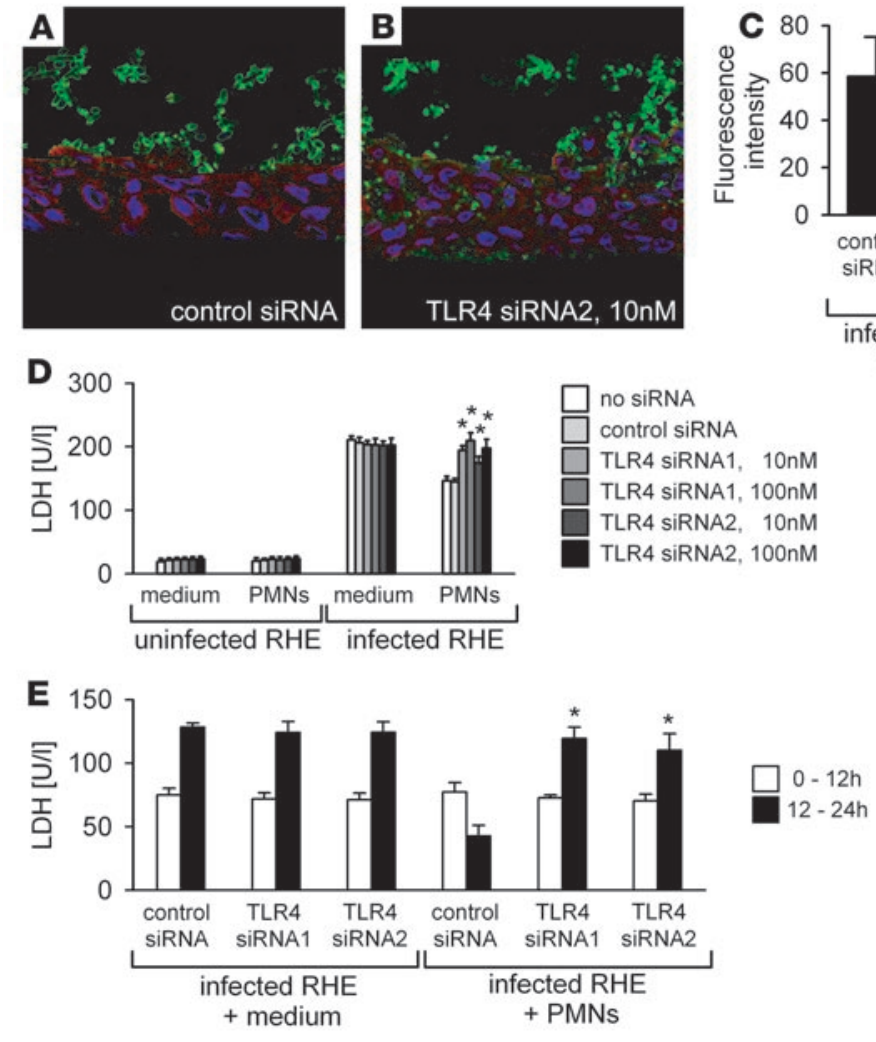

out PMN phagocytosis of invading pathogens and does not even require physical PMN-epithelial cell interactions. Rather, PMNs protected the oral mucosa indirectly through the upregulation of epithelial TLR4, which in turn directly orchestrated the protective response against fungal infection. This protective phenotype was dependent upon the 3-way communication among epithelium, C. albicans, and PMNs, because TLR4 upregulation was not evident in the absence of PMNs or C. albicans and protection from cell injury was not observed without PMNs or TLR4 upregulation. This adds strength to recent observations that epithelial TLRs are involved in protecting mucosal surfaces from cell injury $(6,29,30)$. To our knowledge, this is the first description of such a novel TLRassociated communication mechanism between 2 innate cells at the mucosal surface and implies an extra level of complexity in the PMN-mediated protective response against Candida infections, which might also apply to other fungal or bacterial pathogens.

Although oral epithelial cells express TLRs, no studies have yet demonstrated TLR upregulation upon stimulation with C. albicans. Previously, heat-killed C. albicans cells failed to modulate epithelial TLR expression (13). Similarly, in this study, both heat-killed (data not shown) and viable C. albicans cells were unable to upregulate epithelial TLR expression in the absence of PMNs, despite the fungus causing clear signs of mucosal damage (Figures 1 and 2). With regard to commensal organisms, it has been suggested that rapid responsiveness by epithelial TLRs may create the danger of an immune overreaction (31). Thus, one possible explanation for the lack of direct TLR upregulation by C. albicans may be because the fungus is usually a harmless colonizer of oral mucosal surfaces in approximately $40 \%$ of healthy individuals (32) and may even actively downregulate epithelial responses by unknown mechanisms (our unpublished observations). In addition, during the

\section{Figure 6}

Knockdown of epithelial TLR4 by siRNA abrogates the protective phenotype. (A and B) Confocal microscopy of TLR4 expression in the RHE. C. albicans infection of the RHE and supplementation with PMNs showed strong epithelial TLR4 expression $24 \mathrm{~h}$ after transfection with control siRNA (A), while decreased TLR4 expression associated with increased fungal invasion was evident after transfection with $10 \mathrm{nM}$ TLR4 siRNA2 (B). Original magnification, $\times 40$. (C) Mean fluorescence intensity of TLR4 confirmed reduced expression. Data are representative of 3 independent experiments. (D) Release of LDH by epithelial cells $24 \mathrm{~h}$ after infection (or not) with $\mathrm{C}$. albicans in the presence and absence of PMNs (added after $12 \mathrm{~h})(n=6)$. (E) LDH values before $(0-12 \mathrm{~h})$ and after (12-24 h) addition of PMNs to C. albicans-infected RHE model $(n=4)$. ${ }^{*} P<0.05$, 2-tailed paired Student's $t$ test.

carrier state, it would serve little purpose for the host to activate a TLR-mediated inflammatory response when it is not required.

One mechanism to deal with possible overreactive TLR-immune responses to commensal organisms (i.e., colonizing oral microbes such as C. albicans) is to reduce epithelial cell responsiveness to repeated TLR signaling during prolonged exposure (2). This mechanism was investigated in myeloid differentiation factor 88-deficient (MyD88-/-), TLR4-/-, and TLR2 ${ }^{-/-}$mice, and it was demonstrated that epithelial responsiveness to TLR was not irretrievably diminished and that robust TLR signaling via commensal bacteria during intestinal inflammation protected murine epithelial cells from injury (6). Together with similar murine studies (29), our present data indicate that in addition to their function in host defense, TLRs also contribute to maintenance of epithelial homeostasis and protection from cell injury. Our data support a direct role for TLR4 in protection against epithelial cell injury, because blocking TLR4 with neutralizing antibodies or knockdown of TLR4 mRNA using RNA interference could both override the protective influence of PMNs and restore C. albicans-induced cell damage.

In their study, Rakoff-Nahoum and coworkers (6) also concluded that protection from intestinal epithelial cell injury was not caused by the infiltration of immune cells and excluded a possible role for PMNs in the protective response. Our data, on the other hand, suggest a central role for PMNs in protecting the oral epithelium from fungal-induced cell injury via a process that is independent of PMN infiltration, migration, or direct cell-cell contact with epithelial cells. Therefore, actual PMN infiltration to sites of cell injury may not be an essential factor in inducing protective responses against microbial organisms in vivo, and that PMNs could transmit signals to stimulate epithelial TLR4 expression with the specific remit of maintaining epithelial integrity and homeostasis. Although no direct cell-cell contact is required for this process, we envisage that, in vivo, close proximity of the PMNs with the epithelium would facilitate the induction of epithelial TLR4 expression and protection from cell damage and that PMN infiltration and migration might be beneficial. In MyD88-/- and TLR4-/- mice, such signals from PMNs may fall silent upon the epithelium, resulting in increased cell injury and mortality $(6,29)$. This highlights one advantage of our oral RHE model: the system 

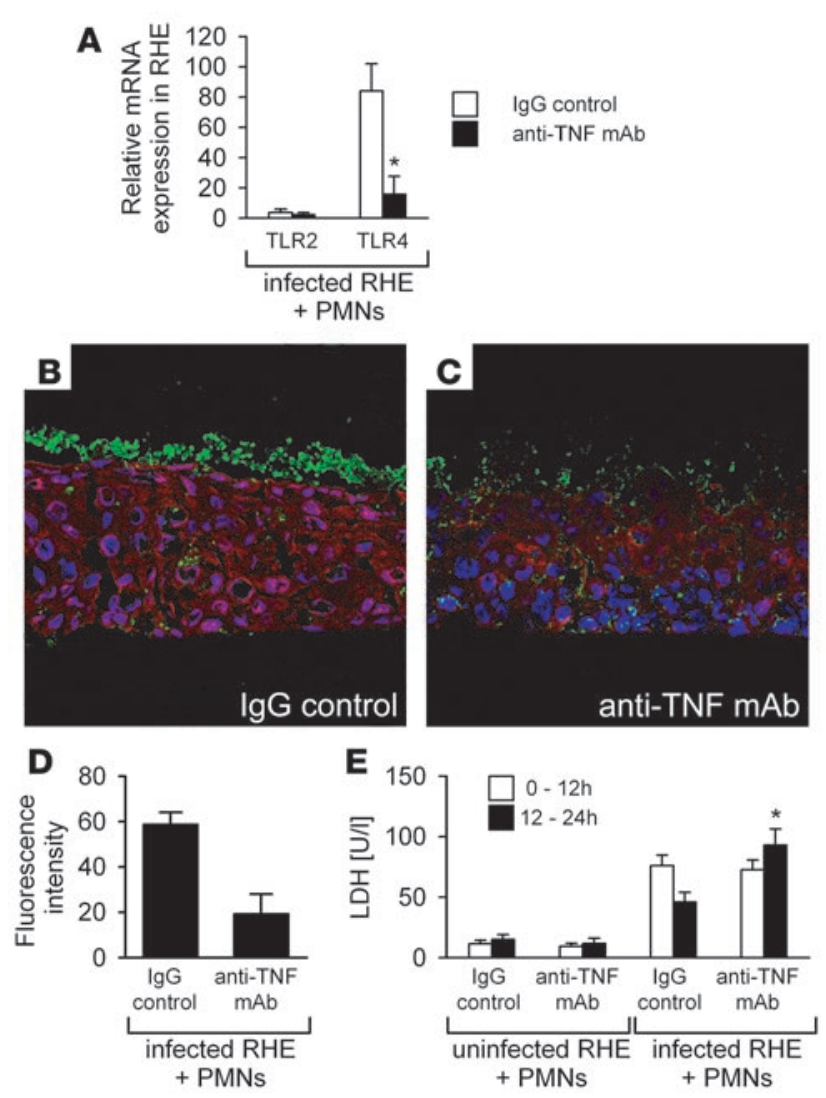

can be supplemented with different immune cell populations, thus allowing specific effects on epithelial TLR expression and protective responses to be scrutinized in detail. In vivo, such analyses are extremely difficult because of the presence of numerous immune cell populations and multiple interacting regulatory pathways.

During oral infection with Candida, a large number of cytokines is secreted by the oral epithelial cells, which maintain a central role in the protection against fungal organisms (20). In general, proinflammatory cytokines (IL- $1 \alpha$, IL- $1 \beta$, IL- 6 , IL- 8 , TNF- $\alpha$, GM-CSF, and others) regulate leukocyte trafficking and/or activate a strong antifungal response by these cells. In the oral RHE model, none of these cytokines alone appeared to have an essential function in host defense, even in the presence of PMNs. Rather, these cytokines appear crucial for the activation of PMNs and/or are released from PMNs, which in turn results in upregulation of epithelial TLR4 and protection from fungal invasion. TNF- $\alpha$ showed the strongest effect, which confirms the important role of this cytokine in host defense against opportunistic fungal infections (33). Absence of this cytokine strongly impairs neutrophil recruitment and effective phagocytosis of C. albicans (34). We also demonstrate that TNF- $\alpha$ is critical for clearance of fungal infections without the contribution of PMN phagocytic activity. Together with the previous finding that TNF- $\alpha$-stimulated PMNs enhance NF- $\mathrm{BB}$ signaling via TLR4 in endothelial cells $(30,35)$, the present study provides what we believe to be novel insights into the molecular mechanisms of TNF- $\alpha$ (and other proinflammatory cytokines) function at the interface between epithelial cells and PMNs. In antibody blocking and siRNA experiments, we observed a statistically significant reduction in IL- 8 secretion in the presence of PMNs when compared with control antibodies and siRNAs. How-

\section{Figure 7}

Neutralization of TNF- $\alpha$ release reduces TLR4 expression and restores fungal invasion. (A) TLR4 mRNA expression was strongly decreased after incubation of the oral RHE and PMNs with anti-TNF- $\alpha$ neutralizing mAbs. Values are normalized to YWHAZ and G6PD and relative to uninfected RHE without PMNs (control, assigned as 1.0). (B and C) Confocal microscopy of TLR4 expression in the infected RHE in the presence of PMNs showed prominent epithelial TLR4 expression at $24 \mathrm{~h}$ after treatment with IgG control antibody (B), while decreased TLR4 expression and increased fungal invasion was evident after incubation with anti-TNF- $\alpha$ mAbs, particularly in the basal side of the epithelium (C). Original magnification, $\times 40$. (D) Reduced mean fluorescence intensity was observed after neutralization of TNF- $\alpha$. Data are representative of 3 independent experiments. (E) Release of LDH by epithelial cells $24 \mathrm{~h}$ after infection (or not) with $C$. albicans in the presence of PMNs (added after $12 \mathrm{~h}$ ). LDH values before (0-12 h) and after (12-24 h) addition of PMNs to $C$. albicans-infected RHE model $(n=3) .{ }^{*} P<0.05$, 2-tailed paired Student's $t$ test.

ever, IL-8 levels were still comparable to those stimulated by C. albicans in the absence of PMNs; and we therefore conclude that IL-8 may not be as biologically significant as TNF- $\alpha$ in controlling the protective response.

Both TLR2 and TLR4 have been implicated in the recognition of C. albicans by immune cells (5). Recently, Netea et al. (36) demonstrated that recognition of surface N-linked mannosyl residues is mediated by mannose receptor and O-linked mannosyl residues by TLR 4 and confirmed previous observations that $\beta$-glucan structures are recognized by TLR 2 in collaboration with Dectin-1 (37). Importantly, the surface N-linked and O-linked mannosyl groups were responsible for most of the cytokinestimulating activity in human mononuclear cells or murine macrophages. In our experiments, stimulation of the oral RHE with heat-killed C. albicans yeast cells, like with viable cells, led to strong upregulation of TLR4 but only a slight increase in TLR2 mRNA, and only in the presence of PMNs, despite the fact that more $\beta$-glucan structures are exposed in the cell wall of heatkilled C. albicans (38). In addition, we demonstrated that blocking of TLR4 restored fungal invasion, whereas no effect was seen after neutralization of TLR2. These findings are in agreement with Netea et al. (36), who observed no difference in recognition of mannans in viable and heat-killed C. albicans, and further indicate that $\alpha$-linked mannan may be an important fungal structure recognized not only by monocytes but also by epithelial cells during host defense in experimental oral candidiasis.

Our data do not exclude the possibility that C. albicans could use non-TLR pattern recognition receptors or TLRs other than TLR4 to initially stimulate cytokine responses. Indeed, TLR3 and not TLR4 is thought to be the most potent inducer of IL-8 from epithelial cells (39). However, to date, no data exist to suggest that C. albicans can activate TLRs other than TLR2 and TLR4, and the present study showed that TLR2 was not involved in the protective process against mucosal Candida infection.

Taken together, our results suggest that the PMN-mediated TLR4-dependent protective mechanism probably occurs in 3 separate phases, which may not necessarily be mutually exclusive. First, in response to C. albicans, the oral epithelium is primed and secretes chemokines and cytokines that recruit and stimulate PMNs at the site of infection. Second, after recruitment, several cytokines, especially TNF- $\alpha$, are directly involved in initiating the subsequent PMN-mediated upregulation of epithelial TLR4 via a process that 
does not require PMN infiltration of the mucosal tissues. Third, epithelial TLR4 directly protects the oral mucosa from fungal invasion and cell injury in an as-yet unidentified manner. Given the vital role of PMNs in the clearance of many fungal and bacterial infections at epithelial surfaces, our data provide insight into a potentially crucial mechanism of innate protection in mucosal tissues and thus how microbial infections could be managed and controlled.

\section{Methods}

Strains, media, and growth conditions. C. albicans wild-type strain SC5314 was used (40). For the infection of the oral RHE and for interaction studies with PMNs, C. albicans was grown on Sabouraud's dextrose agar (Difco) followed by 2 precultures in $10 \mathrm{ml} \mathrm{YPG} \mathrm{(1 \%} \mathrm{yeast} \mathrm{extract,} 2 \%$ peptone, $2 \%$ glucose) medium (Difco), first for $16 \mathrm{~h}$ at $25^{\circ} \mathrm{C}$ and then for $24 \mathrm{~h}$ at $37^{\circ} \mathrm{C}$ through orbital shaking. Heat-inactivated C. albicans cells were prepared as described previously (22).

Model of oral RHE and supplementation with PMNs. Well-established models of oral RHE, based on the buccal epithelial carcinoma cell line TR146, were supplied by SkinEthic Laboratories (41). Infection of oral RHE and isolation of PMNs was performed as described previously $(19,23)$. Briefly, oral RHE samples were incubated with or without $2 \times 10^{6}$ C. albicans SC5314 cells in $50 \mu \mathrm{PBS}$ for 12 and $24 \mathrm{~h}$ at $37^{\circ} \mathrm{C}$ with $5 \% \mathrm{CO}_{2}$ at $100 \%$ humidity. For experiments with PMNs, oral RHE samples were preinfected with C. albicans for 6 or $12 \mathrm{~h}$ and then inverted. PMN cells, at $2 \times 10^{6}$ in $50 \mu \mathrm{l}$ RPMI 1640 medium plus 10\% FCS, were then added directly to the basal side of the polycarbonate filter. The filter prevents cell-cell contact and PMN migration, but allows soluble factors to pass through. After PMN addition, all samples were incubated for a further 6 or $12 \mathrm{~h}$. Control experiments included C. albicans-infected and uninfected oral RHE without PMNs, uninfected oral RHE with PMNs, and PMNs incubated with C. albicans in the absence of oral RHE. Culture medium was added to the basal side of the inverted samples every $60 \mathrm{~min}$ to prevent dehydration and to feed the oral RHE and PMNs. All experiments were performed in the absence of antibiotics and antimycotics. No endotoxin (i.e., LPS) contamination was evident by use of a limulus amebocyte lysate-based kinetic assay (QCL-1000; Cambrex BioScience) or polymyxin B (Sigma-Aldrich).

Epithelial cell damage assay. In all experiments, the release of $\mathrm{LDH}$ from epithelial cells into the surrounding medium at 12 and $24 \mathrm{~h}$ was used as a measure of epithelial cell damage. LDH activity was analyzed spectrophotometrically as described previously (19) and presented as $\mathrm{U} / 1$ at $37^{\circ} \mathrm{C}$.

$R N A$ isolation and quantitative RT-PCR. Total RNA was isolated from oral RHE samples as described previously (19). RNA was reverse transcribed and amplified with the following primers: TLR1, 5'-AACCCATTCCGCAGTACTCCA-3' and 5'-AAGGCCACGTTTGCTCTTTTC-3'; TLR2, 5'-GGAGGCTGCATATTCCAAGG-3' and 5'-GCCAGGCATCCTCACAGG-3'; TLR3, 5' -ACAACTTAGCACGGCTCTGGA-3' and $5^{\prime}$-ACCTCAACTGGGATCTCGTCA-3'; TLR4, 5'-AGTTTCCTGCAATGGATCAAGG-3' and 5'-CTGCTTATCTGAAGGTGTTGCAC-3'; TLR5, $5^{\prime}$-CGAACCTGGAGACAGGAAAA- $3^{\prime}$ and $5^{\prime}$-TCTCCCATGATCCTCGTTGT-3'; TLR6, 5'-CCCATTCCACAGAACAGCAT-3' and 5'-ATAAGTCCGCTGCGTCATGA-3'; TLR7, 5'-TGGAAATTGCCCTCGTTGTT-3' and 5'-GTCAGCGCATCAAAAGCATT-3'; TLR8, 5'-CTTCGATACCTAAACCTCTCTAGCAC-3' and 5'-AAGATCCAGCACCTTCAGATGA-3'; TLR9, 5' -AGTCAATGGCTCCCAGTTCCT-3' and 5'-CGTGAATGAGTGCTCGTGGTA-3'; TLR10, 5'-TGTTATGACAGCAGAGGGTGATG-3' and $5^{\prime}$-GAGTTGAAAAAGGAGGTTATAGGATAAATC-3'; GM-CSF, $5^{\prime}$-TCTCAGAAATGTTTGACCTCCA-3' and 5'-GCCCTTGAGCTTGGTGAG-3'; IL-8, 5'-CAAGAGCCAGGAAGAAACCA-3' and 5'-GTCCACTCTCAATCACTCTCAG-3'; G6PD, 5'-ATCGACCACTACCTGGGCAA- ${ }^{\prime}$ and 5'-TTCTGCATCACGTCCCGGA-3'; YWHAZ, 5'-AGACGGAAGGTGCT-
GAGAAA-3' and 5'-GAAGCATTGGGGATCAAGAA-3'. All PCR variables were optimized for each primer pair. Both standard and experimental samples were performed in triplicate, and the mean threshold cycle $(\mathrm{Ct})$ reading was used. Fold difference in TLR1-TLR10 gene expression was normalized to the housekeeping genes YWHAZ and G6PD, because detailed preliminary investigations using 10 different housekeeping genes (ALDOA, B2M, G6PD, GAPDH, HMBS, POLR2A, SDHA, TBP, UBC, and YWHAZ) indicated that these genes showed the most constant level of expression.

Inhibition of epithelial TLR2 and TLR4 using neutralizing antibodies. Epithelial TLR2 and TLR4 were inhibited using functional-grade anti-human TLR2specific neutralizing mAb TL2.1 and TLR4-specific neutralizing mAb HTA-125. An IgG2a isotype antibody was used as a control (eBioscience). TLR2, TLR4, and isotype control antibodies $(20 \mu \mathrm{g} / \mathrm{ml})$ were added to RHE cultures $1 \mathrm{~h}$ prior to C. albicans infection.

Knockdown of TLR4 expression using RNA interference. Two different siRNA duplexes were synthesized (Qiagen) to knock down TLR4 expression (TLR4 siRNA1, 5'-CGAUGAUAUUAUUGACUUAdTdT-3' and 5'-UAAGUCAAUAAUAUCAUCGdAdG-3'; TLR4 siRNA2, 5'-CAAAGUUAUUGCCUACUAAdTdT- ${ }^{\prime}$ and $5^{\prime}$-UUAGUAGGCAAUAACUUUGdGdA-3'). As a control, a nonsilencing siRNA duplex was used ( $5^{\prime}$-UUCUCCGAACGUGUCACGUdTT-3' and 5'-ACGUGACACGUUCGGAGAAdTT-3'; Qiagen). TLR4 and control siRNA stock solutions were prepared according to the manufacturer's instructions (HiPerFect; Qiagen). Oral RHE samples were incubated with siRNA for $24 \mathrm{~h}$ prior to infection.

Epithelial cytokine production and inhibition. Culture medium was collected at the conclusion of the oral RHE experiments, usually $24 \mathrm{~h}$, and assayed for G-CSF, GM-CSF, IL-1 $\alpha$, IL-1 $\beta$, IL-2, IL-4, IL-6, IL-8, IL-12, MCP-1, MIP-1 $\beta$, and TNF- $\alpha$, using commercially available ELISA kits (DuoSet; R\&D Systems). For TLR4 blocking experiments, TLR4 neutralizing antibody and an IgG2a isotype control was added $1 \mathrm{~h}$ prior to the addition of C. albicans. At the conclusion of the experiment (24 h), IL-8 and GM-CSF protein levels were quantified in the culture supernatant. For cytokine blocking experiments, GM-CSF $(10 \mu \mathrm{g} / \mathrm{ml})$, IL-1 $\alpha(0.1 \mu \mathrm{g} / \mathrm{ml})$, IL-1 $\beta$ $(0.1 \mu \mathrm{g} / \mathrm{ml})$, IL-6 $(1 \mu \mathrm{g} / \mathrm{ml})$, IL-8 $(1 \mu \mathrm{g} / \mathrm{ml})$, MCP-1 $(10 \mu \mathrm{g} / \mathrm{ml})$, MIP-1 $\beta$ $(1 \mu \mathrm{g} / \mathrm{ml})$, and TNF- $\alpha(1 \mu \mathrm{g} / \mathrm{ml})$ blocking antibodies and corresponding IgG isotype controls (R\&D Systems) were added to the RHE $1 \mathrm{~h}$ prior to the addition of C. albicans. In some experiments, PMNs were treated with cytokine-blocking antibodies or IgG isotype controls for $1 \mathrm{~h}$ before supplementation to the RHE.

Light, confocal, and immunoelectron microscopy. Light microscopical studies were performed as previously described using paraffin-embedded oral RHE specimens $(19,22)$. For confocal microscopy, oral RHE was cryofixed in liquid nitrogen, and $5-\mu \mathrm{m}$ sections were placed on silan-coated slides. Sections were fixed in PLP (paraformaldehyde and lysine in PBS) for $2 \mathrm{~min}$, followed by incubation with PBS for 5 min, PBS/BSA (0.1\%) plus Tween 20 (0.1\%) for $10 \mathrm{~min}$, and PBS plus $10 \%$ donkey serum for $30 \mathrm{~min}$ at room temperature. Anti-TLR4 polyclonal rabbit sc-10741 antibodies (1:40; Santa Cruz Biotechnology) and human anti-C. albicans serum (1:60; Virion $\backslash$ Serion) were added overnight at $4{ }^{\circ} \mathrm{C}$. Sections were then incubated with donkey anti-rabbit-Cy2 (1:100; Dianova) and donkey anti-human-Cy3 (1:500; Dianova) for $60 \mathrm{~min}$. All nuclei were stained with TOPRO (Invitrogen). All washing and antibody addition steps were performed with a combination of PBS, BSA, and Tween. The sections were analyzed with a confocal laser scanning microscope (Leica TCS SP; Leica Microsystems) at $\times 40$ and $\times 250$ magnification. To determine the relative levels of TLR4 protein expression, fluorescence intensity measurements were performed on the confocal images using the Leica PowerScan software. Representative lines denoting region of interest were drawn across each section, and the mean pixel intensity above background was obtained. For electron microscopy, oral RHE samples were fixed and embedded in Lowicryl (Polysciences). Ultrathin 
sections $(50 \mathrm{~nm})$ were mounted on Formvar-coated nickel grids and incubated with rabbit anti-TLR4 antibody followed by $10 \mathrm{~nm}$ gold-conjugated goat anti-rabbit IgG (Immunogold reagents; Auroprobe EM). In control samples, the primary Ab was omitted. Grids were counterstained with uranyl acetate and lead citrate and examined using a Zeiss 109 transmission electron microscope at $\times 3,000$ and $\times 20,000$ magnification (Zeiss).

Statistics. All experiments were performed at least 3 times and revealed comparable results. Results are presented as mean \pm SD. Statistical significance was determined using the 2 -tailed paired Student's $t$ test. A $P$ value of 0.05 or less was considered significant.

\section{Acknowledgments}

The authors would like to thank Anwar Tappuni for the collection of patient samples and David Moyes, Christiane Nüsslein-Volhard, and Martin Röcken for helpful discussions. We thank Birgit Fehrenbacher, Renate Nordin, Helga Möller, and Hannelore Bischof (University of Tübingen) for excellent technical assistance. M. Schaller and G. Weindl were supported by the Deutsche Forschungsgemeinschaft
(Sch 897/1 and Sch 897/3), and G. Weindl was supported by GK 303, "Infection and Immunity." B. Hube was supported by the Deutsche Forschungsgemeinschaft (Hu 528/10), and J. Naglik was supported by NIDCR grant R01 DE017514-01 and a personal Wellcome Trust Value in People (VIP) award. T. Biedermann and S. Kaesler were supported by the Deutsche Forschungsgemeinschaft (Bi 696/3-1 and SFB 685 A6, respectively).

Received for publication February 3, 2006, and accepted in revised form August 30, 2007.

Address correspondence to: M. Schaller, Department of Dermatology, Eberhard Karls University Tübingen, Liebermeisterstrasse 25, D-72076 Tübingen, Germany. Phone: 49-7071-29-84555; Fax: 49-7071-29-5113; E-mail: Martin.Schaller@med.uni-tuebingen.de.

Günther Weindl and Julian R. Naglik contributed equally to this work.
1. Godaly, G., et al. 2001. Neutrophil recruitment, chemokine receptors, and resistance to mucosal infection. J. Leukoc. Biol. 69:899-906.

2. Backhed, F., and Hornef, M. 2003. Toll-like receptor 4-mediated signaling by epithelial surfaces: necessity or threat? Microbes Infect. 5:951-959.

3. Tjabringa, G.S., et al. 2005. Host defense effector molecules in mucosal secretions. FEMS. Immunol. Med. Microbiol. 45:151-158.

4. Takeda, K., Kaisho, T., and Akira, S. 2003. Toll-like receptors. Annu. Rev. Immunol. 21:335-376.

5. Roeder, A., Kirschning, C.J., Rupec, R.A., Schaller, M., and Korting, H.C. 2004. Toll-like receptors and innate antifungal responses. Trends Microbiol. 12:44-49.

6. Rakoff-Nahoum, S., et al. 2004. Recognition of commensal microflora by toll-like receptors is required for intestinal homeostasis. Cell. 118:229-241.

7. Viscoli, C., et al. 1999. Candidemia in cancer patients: a prospective, multicenter surveillance study by the Invasive Fungal Infection Group (IFIG) of the European Organization for Research and Treatment of Cancer (EORTC). Clin. Infect. Dis. 28:1071-1079.

8. Tortorano, A.M., et al. 2004. Epidemiology of candidaemia in Europe: results of 28-month European Confederation of Medical Mycology (ECMM) hospital-based surveillance study. Eur.J. Clin. Microbiol. Infect. Dis. 23:317-322.

9. Ozinsky, A., et al. 2000. The repertoire for pattern recognition of pathogens by the innate immune system is defined by cooperation between toll-like receptors. Proc. Natl. Acad. Sci. U. S. A. 97:13766-13771.

10. Netea, M.G., et al. 2002. The role of toll-like receptor (TLR) 2 and TLR4 in the host defense against disseminated candidiasis. J. Infect. Dis. 185:1483-1489.

11. Mahanonda, R., and Pichyangkul, S. 2007. Toll-like receptors and their role in periodontal health and disease. Periodontol. 2000. 43:41-55.

12. Sugawara, Y., et al. 2006. Toll-like receptors, NOD1, and NOD2 in oral epithelial cells. J. Dent. Res. 85:524-529.

13. Pivarcsi, A., et al. 2003. Expression and function of Toll-like receptors 2 and 4 in human keratinocytes. Int. Immunol. 15:721-730.

14. Reaves, T.A., Chin, A.C., and Parkos, C.A. 2005. Neutrophil transepithelial migration: role of tolllike receptors in mucosal inflammation. Mem. Inst. Oswaldo Cruz. 100(Suppl. 1):191-198.

15. Liu, Y., et al. 2004. Regulation of leukocyte transmigration: cell surface interactions and signaling events. J. Immunol. 172:7-13.

16. Koyama, S., et al. 1991. Endotoxin stimulates bron- chial epithelial cells to release chemotactic factors for neutrophils. A potential mechanism for neutrophil recruitment, cytotoxicity, and inhibition of proliferation in bronchial inflammation. J. Immunol. 147:4293-4301.

17. Nusrat, A., et al. 1997. Neutrophil migration across model intestinal epithelia: monolayer disruption and subsequent events in epithelial repair. Gastroenterology. 113:1489-1500.

18. Fidel, P.L., Jr. 2002. Distinct protective host defenses against oral and vaginal candidiasis. Med. Mycol. 40:359-375.

19. Schaller, M., et al. 2004. Polymorphonuclear leukocytes (PMNs) induce protective Th1-type cytokine epithelial responses in an in vitro model of oral candidosis. Microbiology. 150:2807-2813.

20. Dongari-Bagtzoglou, A., and Fidel, P.L., Jr. 2005. The host cytokine responses and protective immunity in oropharyngeal candidiasis. J. Dent. Res. 84:966-977.

21. Schaller, M., Schafer, W., Korting, H.C., and Hube, B. 1998. Differential expression of secreted aspartyl proteinases in a model of human oral candidosis and in patient samples from the oral cavity. Mol. Microbiol. 29:605-615.

22. Schaller, M., et al. 2002. Infection of human oral epithelia with Candida species induces cytokine expression correlated to the degree of virulence. J. Invest. Dermatol. 118:652-657.

23. Schaller, M., Zakikhany, K., Naglik, J.R., Weindl, G., and Hube, B. 2006. Models of oral and vaginal candidiasis based on in vitro reconstituted human epithelia. Nat. Protoc. 1:2767-2773.

24. Hayashi, F., Means, T.K., and Luster, A.D. 2003. Toll-like receptors stimulate human neutrophil function. Blood. 102:2660-2669.

25. Challacombe, S.J. 1994. Immunologic aspects of oral candidiasis. Oral Surg. Oral Med. Oral Pathol. 78:202-210.

26. Eversole, L.R., et al. 1997. Oral keratinocyte immune responses in HIV-associated candidiasis. Oral Surg. Oral Med. Oral Pathol. Oral Radiol. Endod. 84:372-380.

27. Hornef, M.W., et al. 2002. Toll-like receptor 4 resides in the Golgi apparatus and colocalizes with internalized lipopolysaccharide in intestinal epithelial cells. J. Exp. Med. 195:559-570.

28. Yamashiro, S., et al. 2001. Phenotypic and functional change of cytokine-activated neutrophils: inflammatory neutrophils are heterogeneous and enhance adaptive immune responses. J. Leukoc. Biol. 69:698-704.

29. Fukata, M., et al. 2005. Toll-like receptor-4 is required for intestinal response to epithelial injury and limiting bacterial translocation in a murine model of acute colitis. Am. J. Physiol. Gastrointest. Liver Physiol. 288:G1055-G1065.

30. Fan, J., Frey, R.S., and Malik, A.B. 2003. TLR4 signaling induces TLR2 expression in endothelial cells via neutrophil NADPH oxidase. J. Clin. Invest. 112:1234-1243. doi:10.1172/JCI200318696.

31. Strober, W. 2004. Epithelial cells pay a Toll for protection. Nat. Med. 10:898-900.

32. Arendorf, T.M., and Walker, D.M. 1979. Oral candidal populations in health and disease. Br. Dent.J. 147:267-272.

33. Filler, S.G., Yeaman, M.R., and Sheppard, D.C. 2005. Tumor necrosis factor inhibition and invasive fungal infections. Clin. Infect. Dis. 41(Suppl. 3):S208-S212.

34. Netea, M.G., et al. 1999. Increased susceptibility of TNF-alpha lymphotoxin-alpha double knockout mice to systemic candidiasis through impaired recruitment of neutrophils and phagocytosis of Candida albicans. J. Immunol. 163:1498-1505.

35. Fan, J., Frey, R.S., Rahman, A., and Malik, A.B. 2002. Role of neutrophil NADPH oxidase in the mechanism of tumor necrosis factor-alpha -induced NFkappa $\mathrm{B}$ activation and intercellular adhesion molecule-1 expression in endothelial cells. J. Biol. Chem. 277:3404-3411.

36. Netea, M.G., et al. 2006. Immune sensing of Candida albicans requires cooperative recognition of mannans and glucans by lectin and Toll-like receptors. J. Clin. Invest. 116:1642-1650. doi:10.1172/JCI27114.

37. Gantner, B.N., Simmons, R.M., Canavera, S.J., Akira, S., and Underhill, D.M. 2003. Collaborative induction of inflammatory responses by dectin-1 and Toll-like receptor 2. J. Exp. Med. 197:1107-1117.

38. Gantner, B.N., Simmons, R.M., and Underhill, D.M. 2005. Dectin-1 mediates macrophage recognition of Candida albicans yeast but not filaments. EMBO J. 24:1277-1286.

39. Kollisch, G., et al. 2005. Various members of the Toll-like receptor family contribute to the innate immune response of human epidermal keratinocytes. Immunology. 114:531-541.

40. Gillum, A.M., Tsay, E.Y., and Kirsch, D.R. 1984. Isolation of the Candida albicans gene for orotidine5 -phosphate decarboxylase by complementation of S. cerevisiae ura3 and E. coli pyrF mutations. Mol. Gen. Genet. 198:179-182.

41. Rupniak, H.T., et al. 1985. Characteristics of four new human cell lines derived from squamous cell carcinomas of the head and neck. J. Natl. Cancer Inst. 75:621-635. 